\title{
Mutual inductance of two coaxial rectangular PCB coils incorporating magnetic layer
}

\author{
Salah-Eddine Bendimerad ${ }^{1 *}$, Selma Baghli ${ }^{2}$, \\ Abdelghani Ayad ${ }^{3}$, Amar Tilmatine ${ }^{1}$
}

\begin{abstract}
The wireless connection distance between the transmission and reception coils of printed circuit boards (PCB) influences the mutual inductance and affects circuit performance. In this study, the mutual inductance $M$ of $\mathrm{PCB}$ coils was investigated, and two analytical methods were presented for calculating the mutual inductance between two coaxial rectangular planar PCB coils incorporating magnetic layer. The results were acquired through calculations by using the Neumann integral and Biot-Savart methods. The complete integral calculations and detailed demonstrations of the two methods are presented. The obtained formulas were introduced in some examples of coils with different number of turns. The analytical and experimental results were compared, and a strong agreement between them was observed.
\end{abstract}

K e y w ords: mutual inductance, rectangular planar PCB coil, ferromagnetic layer, Neumann formula, Biot-Savart law

\section{Introduction}

Printed circuit board (PCB) coils are an essential part of modern electrical equipment and are utilised in diverse domains. In the 2010s, wireless power transfer (WPT) has been increasingly used. WPT presents several applications in many fields, such as implantable medical devices, noncontact charging batteries of electric cars, and wireless chargers for smartphones. Inductive coupling is the most commonly used method for energy transfer. Generally, two coaxial inductors are employed for this coupling. Moreover, conventional or planar PCB coils can be used for inductive coupling. Planar PCB coils are inexpensive, robust and can be produced on a large scale. Moreover, depending on applications, these coils can be manufactured on hard or flexible substrates [1-3].

The increase in the mutual inductance, also increases the coupling efficiency of two Transmission and Reception coils (T- and R-coils) [4]. The technique of embedding a magnetic layer under the T-coil, also greatly increases the value of the mutual inductance $[5,6]$. In this work, an approach is proposed for a fast calculation of the mutual inductance incorporating a ferromagnetic material into the inductors as a part of their structure. A study is to investigate a mutual inductance of two rectangular printed spiral coils, by an analytical methods for the calculation, from the Neumann integral and Biot-Savart law with a magnetic layer.

We present the mathematical modelling of the mutual inductance from the Neumann integral and Biot-Savart Law, and compare the calculation results of the two methods with experimental measurement.

\section{Mathematical modelling}

A basic configuration of a $\mathrm{PCB}$ rectangular spiral inductors above a magnetic layer is considered. The metallic rectangular-spiral is assumed to have zero thickness, filamentary and to be located in free space. Although the FR-4 material (epoxy glass substrates) are taken as free space in our configuration, because his permeability is very close to unity. This modelization is based on the assumption that the permeability of the magnetic layer is isotropic and infinite. The configuration is shown in Fig. 1.

The distance between the rectangular spiral and the magnetic layer is denoted by $d_{0}$. To facilitate the calculations, the following replacement and additional assumptions, are used as described below. Firstly, the replacement of the rectangular spiral by concentric rectangular rings with the same track width and strip spacing. The number of the concentric square rings is equal to the number of turns of the rectangular spiral. The mutual inductance is calculated by a magnetostatic model, ie, direct currents are used for the modelling.

Based on these assumptions, in the half-space $z<$ $-d_{0}$, a magnetic induction $\boldsymbol{B}$ is supposed to be created by current image at $z=-2 d_{0}$. The magnitude and direction of the image surface-current density are the same as those of the original surface-current density of T-coil in $z=0,[5,6]$.

The calculations are mainly focused on the mutual inductance between the image of coil $\mathrm{T}$ and the coil $\mathrm{R}$.

\footnotetext{
${ }^{1}$ APELEC Laboratory, ${ }^{3}$ ICEPS Laboratory, Electrical Engineering Faculty, Djillali Liabes University of Sidi Bel-Abbes, Algeria, bendimerad.s@gmail.com, ${ }^{2}$ Mathematics Laboratory, Exact Sciences Faculty, Djillali Liabes University of Sidi Bel-Abbes, Algeria
}

https://doi.org/10.2478/jee-2021-0041, Print (till 2015) ISSN 1335-3632, On-line ISSN 1339-309X

(C) This is an open access article licensed under the Creative Commons Attribution-NonCommercial-NoDerivs License (http: //creativecommons.org/licenses/by-nc-nd/3.0/). 


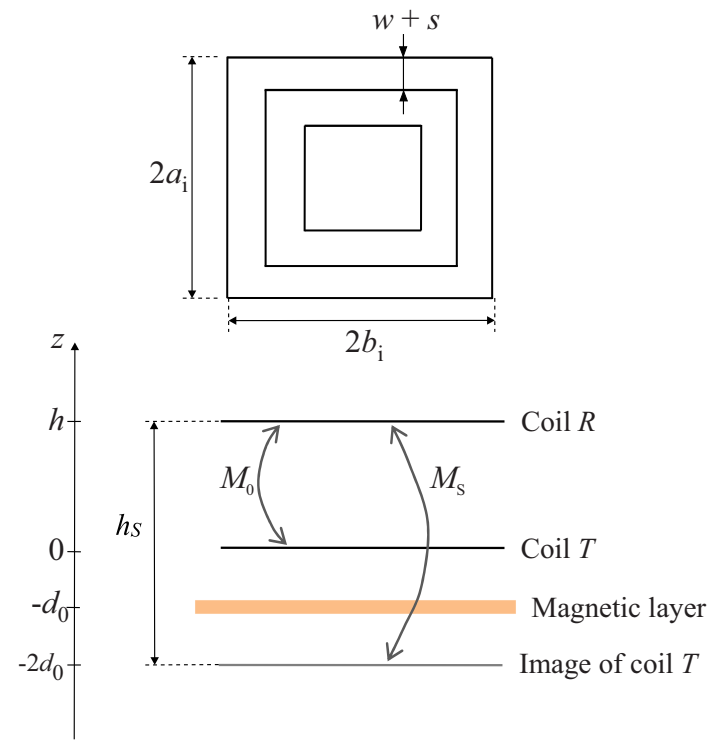

Fig. 1. Configuration of concentric rectangular spiral inductors above a magnetic layer

\subsection{The Neumann formula}

The inductance of circuits constituting straight elements, that is, straights wire joined in parallel or series, can be obtained through summation. The mutual inductance of the overall circuit is the sum of all the pairs of elements. When the distance between wires is larger than the cross-section dimension, the mutual inductance of each pair of wires is the same as that of filaments along the wire axis, irrespective of the wire cross sections. If the circuit comprises many elements, calculations become tedious because of the large number of turns. Therefore, general formulas for the cases derived by this method are important $[7,8]$. The thin copper tracks of PCB coils were first modelled as multiple straight-line filaments $[9,10]$. To commence the calculus, let us consider two straights parallel filaments with the length of $2 a$ and $2 c$ (Fig. 2).

The Neumann formula is used to calculate the mutual inductance of two filaments, [8]

$$
M_{\mathrm{S}}^{\mathrm{N}}=\frac{\mu_{0}}{4 \pi} \iint \frac{\cos \theta \mathrm{d} y \mathrm{~d} y^{\prime}}{r}
$$

where $\mu_{0}$ is the permeability of free space.

The two filaments are parallel; hence, $\theta=0$, or $\cos \theta=$ 1.

The distance between the two filament elements, $\mathrm{d} y$ and $\mathrm{d} y^{\prime}$, is

$$
r=\sqrt{h_{\mathrm{S}}^{2}+\left(y^{\prime}-y\right)^{2}}
$$

where $h_{\mathrm{S}}$ is the lift distance between image of coils $\mathrm{T}$ and coil R. Then the mutual inductance becomes

$$
M_{\mathrm{S}}^{\mathrm{N}}=\frac{\mu_{0}}{4 \pi} \int_{-a}^{+a}\left(\int_{-c}^{+c} \frac{\mathrm{d} y^{\prime}}{\sqrt{h_{\mathrm{S}}^{2}+\left(y^{\prime}-y\right)^{2}}}\right) \mathrm{d} y .
$$

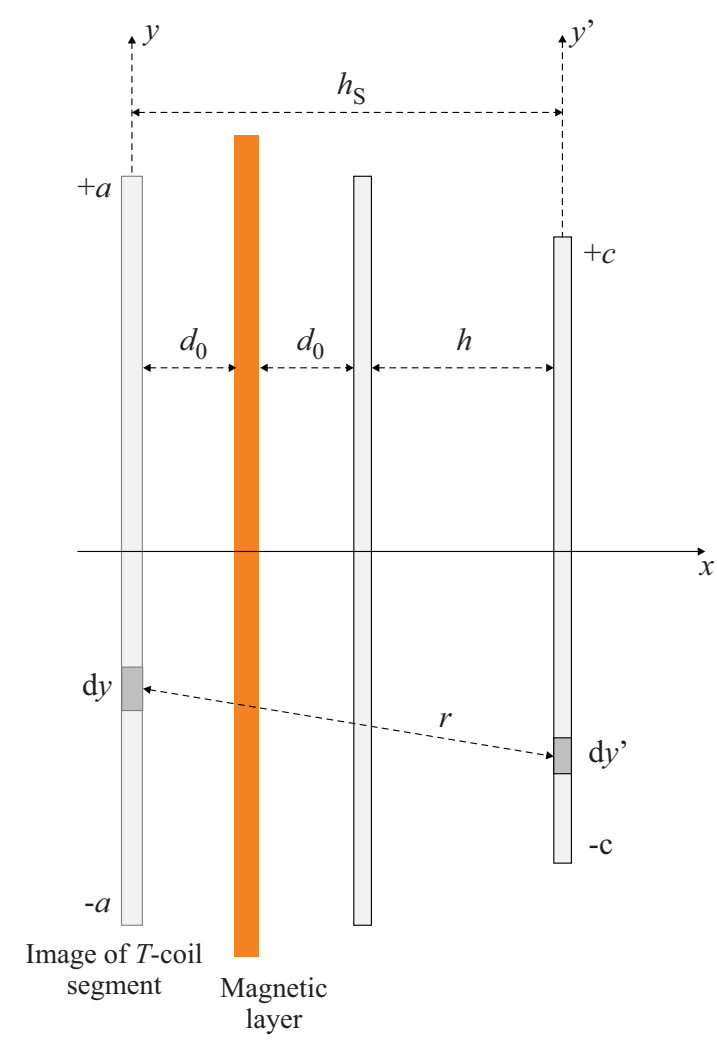

Fig. 2. Two straights parallel filaments considered in the calculations

Using the inverse hyperbolic sinus primitive, we get

$$
M_{\mathrm{S}}^{\mathrm{N}}=\frac{\mu_{0}}{4 \pi} \int_{-a}^{+a}\left[\sinh ^{-1}\left(\frac{y^{\prime}-y}{h_{\mathrm{S}}}\right)^{2}\right]_{-c}^{+c} \mathrm{~d} y .
$$

Consequently,

$M_{\mathrm{S}}^{\mathrm{N}}=\frac{\mu_{0}}{4 \pi} \int_{-a}^{+a} \sinh ^{-1}\left(\frac{c-y}{h_{\mathrm{S}}}\right) \mathrm{d} y+\frac{\mu_{0}}{4 \pi} \int_{-a}^{+a} \sinh ^{-1}\left(\frac{c+y}{h_{\mathrm{S}}}\right) \mathrm{d} y$.

Set

$$
I n t_{1}=\int_{-a}^{+a} \sinh ^{-1}\left(\frac{c-y}{h_{\mathrm{S}}}\right) \mathrm{d} y
$$

and

$$
\operatorname{Int}_{2}=\int_{-a}^{+a} \sinh ^{-1}\left(\frac{c+y}{h_{\mathrm{S}}}\right) \mathrm{d} y
$$

then

$$
M_{\mathrm{S}}^{\mathrm{N}}=\frac{\mu_{0}}{4 \pi}\left(\operatorname{Int}_{1}+\operatorname{Int}_{2}\right) .
$$

First, we calculate $\operatorname{Int}_{1}$

$$
I n t_{1}=h_{\mathrm{S}} \int_{\frac{c-a}{h_{\mathrm{S}}}}^{\frac{(c+a)}{h_{\mathrm{S}}}} \sinh ^{-1} x \mathrm{~d} x .
$$

Then, we obtain

$$
\begin{array}{r}
\text { Int }_{1}=(c+a) \sinh ^{-1}\left(\frac{c+a}{h_{\mathrm{S}}}\right)-(c-h) \sinh ^{-1}\left(\frac{c-a}{h_{\mathrm{S}}}\right)- \\
h_{\mathrm{S}}\left(\sqrt{1+\left(\frac{c+a}{h_{\mathrm{S}}}\right)^{2}}-\sqrt{1+\left(\frac{c-a}{h_{\mathrm{S}}}\right)^{2}}\right) .
\end{array}
$$



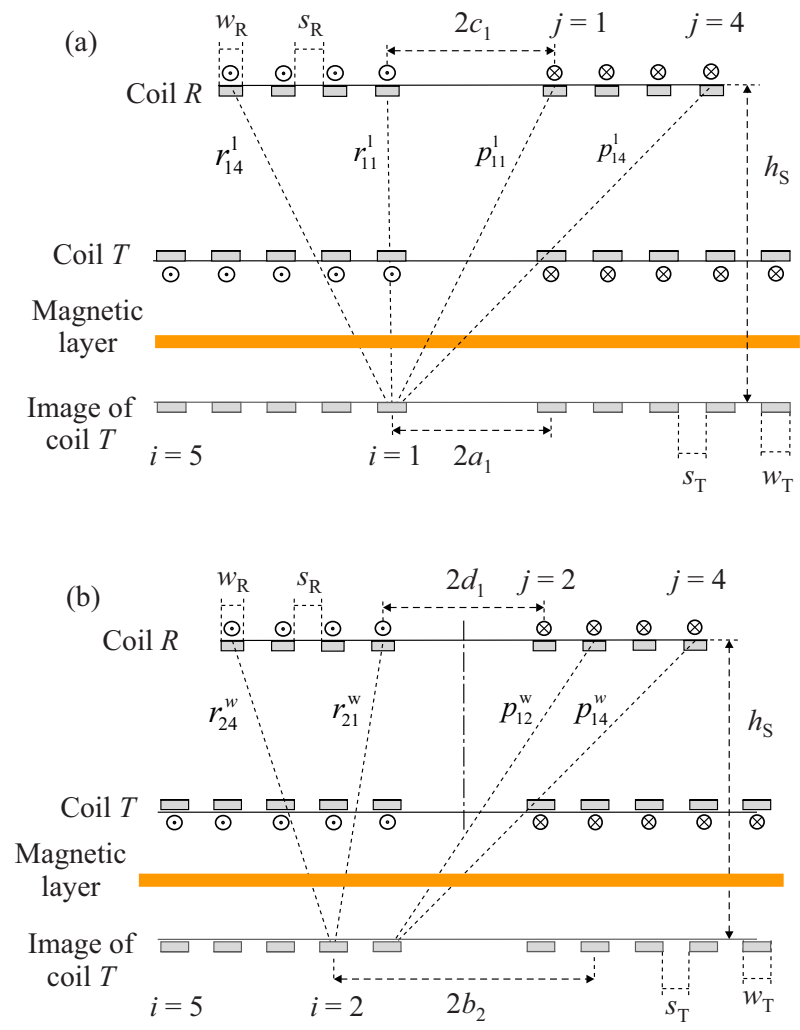

Fig. 3. Different distances between wire segments

Idem, we acquire

$$
\begin{array}{r}
\text { Int }_{2}=(c+a) \sinh ^{-1} \frac{c+a}{h_{\mathrm{S}}}-(c-h) \sinh ^{-1} \frac{c-a}{h_{\mathrm{S}}}- \\
h_{\mathrm{S}}\left(\sqrt{1+\left(\frac{c+a}{h_{\mathrm{S}}}\right)^{2}}-\sqrt{1+\left(\frac{c-a}{h_{\mathrm{S}}}\right)^{2}}\right) .
\end{array}
$$

Thus, the mutual inductance between the two filaments is

$$
\begin{aligned}
M_{\mathrm{S}}^{N} & =\frac{\mu_{0}}{2 \pi}\left[(c+a) \sinh ^{-1}\left(\frac{c+a}{h_{\mathrm{S}}}\right)-h_{\mathrm{S}} \sqrt{1+\left(\frac{c+a}{h_{\mathrm{S}}}\right)^{2}}\right. \\
& \left.-(c-a) \sinh ^{-1}\left(\frac{c-a}{h_{\mathrm{S}}}\right)-h_{\mathrm{S}} \sqrt{1+\left(\frac{c-a}{h_{\mathrm{S}}}\right)^{2}}\right]
\end{aligned}
$$

In our case, to calculate the global mutual inductance between the two PCB rectangular-planar coils, we computed the sum of each mutual inductance between the two segments. The mutual inductances between the two segments with the same and opposite current directions have the positive and negative signs, respectively. The distance between the two segments with the same and opposite current directions are $r_{i j}$ and $p_{i j}$, respectively. For rectangular coils $\mathrm{T}$ and $\mathrm{R}$, the lengths of two sides are $a_{i}$ and $c_{j}$, respectively, and the width of two sides are $b_{i}$ and $d_{j}$, respectively. Let us obtain the general form of distances between the two segments of image of coil $\mathrm{T}$ and coil R; the coils are numbered from inside to outside, Fig. 2.

$$
\begin{gathered}
r_{i j}^{\text {length }}=r_{i j}^{l}=\sqrt{h_{\mathrm{S}}^{2}+\left(\left(a_{i}-c_{j}\right)+(i-1) d_{T}-(j-1) d_{R}\right)^{2}}, \\
r_{i j}^{\text {width }}=r_{i j}^{w}=\sqrt{h_{\mathrm{S}}^{2}+\left(\left(b_{i}-d_{j}\right)+(i-1) d_{T}-(j-1) d_{R}\right)^{2}}, \\
p_{i j}^{\text {length }}=p_{i j}^{l}=\sqrt{h_{\mathrm{S}}^{2}+\left(\left(a_{i}+c_{j}\right)+(i-1) d_{T}-(j-1) d_{R}\right)^{2}}, \\
p_{i j}^{\text {width }}=p_{i j}^{w}=\sqrt{h_{\mathrm{S}}^{2}+\left(\left(b_{i}+d_{j}\right)+(i-1) d_{T}-(j-1) d_{R}\right)^{2}}
\end{gathered}
$$

with $d_{T}=s_{T}+w_{T}$, and $d_{R}=s_{R}+w_{R}$.

Thus, the general form for the mutual inductance of the two segments is

$$
\begin{aligned}
& M^{\mathrm{rl}}=\frac{\mu_{0}}{2 \pi} \times \\
& \sum_{i=1}^{N_{T}} \sum_{j=1}^{N_{R}}\left[\left(c_{j}+a_{i}\right) \sinh ^{-1}\left(\frac{c_{j}+a_{i}}{r_{i j}^{l}}\right)-r_{i j}^{l} \sqrt{1+\left(\frac{c_{j}+a_{i}}{r_{i j}^{l}}\right)^{2}}\right. \\
& \left.-\left(c_{j}-a_{i}\right) \sinh ^{-1}\left(\frac{c_{j}-a_{i}}{r_{i j}^{l}}\right)-r_{i j}^{l} \sqrt{1+\left(\frac{c_{j}-a_{i}}{r_{i j}^{l}}\right)^{2}}\right]
\end{aligned}
$$

for the mutual induction of the side length of the two segments, which have the same current direction.

$$
\begin{aligned}
& M^{\mathrm{rw}}=\frac{\mu_{0}}{2 \pi} \times \\
& \sum_{i=1}^{N_{T}} \sum_{j=1}^{N_{R}}\left[\left(d_{j}+b_{i}\right) \sinh ^{-1}\left(\frac{d_{j}+b_{i}}{r_{i j}^{w}}\right)+r_{i j}^{w} \sqrt{1+\left(\frac{c_{j}+a_{i}}{r_{i j}^{w}}\right)^{2}}\right. \\
& \left.-\left(c_{j}-a_{i}\right) \sinh ^{-1}\left(\frac{d_{j}-b_{i}}{r_{i j}^{w}}\right)+r_{i j}^{w} \sqrt{1+\left(\frac{d_{j}-b_{i}}{r_{i j}^{w}}\right)^{2}}\right], \quad(6)
\end{aligned}
$$

for the mutual induction of the side width of the two segments, which have the same current direction.

For the two segments with the opposite current direction

$$
\begin{aligned}
& M^{\mathrm{pl}}=\frac{\mu_{0}}{2 \pi} \times \\
& \sum_{i=1}^{N_{T}} \sum_{j=1}^{N_{R}}\left[\left(c_{j}+a_{i}\right) \sinh ^{-1}\left(\frac{c_{j}+a_{i}}{p_{i j}^{l}}\right)-p_{i j}^{l} \sqrt{1+\left(\frac{c_{j}+a_{i}}{p_{i j}^{l}}\right)^{2}}\right. \\
& \left.-\left(c_{j}-a_{i}\right) \sinh ^{-1}\left(\frac{c_{j}-a_{i}}{p_{i j}^{l}}\right)+p_{i j}^{l} \sqrt{1+\left(\frac{c_{j}-a_{i}}{p_{i j}^{l}}\right)^{2}}\right],
\end{aligned}
$$

for the mutual induction of the side length of the two segments, which have the opposite current direction.

$$
\begin{aligned}
& M^{\mathrm{pw}}=\frac{\mu_{0}}{2 \pi} \times \\
& \sum_{i=1}^{N_{T}} \sum_{j=1}^{N_{R}}\left[\left(d_{j}+b_{i}\right) \sinh ^{-1}\left(\frac{d_{j}+b_{i}}{p_{i j}^{w}}\right)-p_{i j}^{w} \sqrt{1+\left(\frac{d_{j}+b_{i}}{p_{i j}^{w}}\right)^{2}}\right. \\
& \left.-\left(d_{j}-b_{i}\right) \sinh ^{-1}\left(\frac{d_{j}-b_{i}}{p_{i j}^{w}}\right)+p_{i j}^{w} \sqrt{1+\left(\frac{d_{j}-b_{i}}{p_{i j}^{w}}\right)^{2}}\right]
\end{aligned}
$$


for the mutual induction of the side width two segments, which have the opposite current direction.

By using the Neumann method, the mutual inductance of the image T-coil and the R-coil is

$$
M_{\mathrm{S}}^{\mathrm{N}}=2\left(M^{\mathrm{rl}}+M^{\mathrm{rw}}-M^{\mathrm{pl}}-M^{\mathrm{pw}}\right) .
$$

We acquired the double of each mutual inductance because we used a couple of lengths and widths in each coil.

Finally, the global mutual inductance $M^{\mathrm{N}}$ is equal to the sum of the mutual inductance without magnetic layer $M_{0}^{\mathrm{N}}$, and the mutual inductance of the image of T-coil with R-coil $M_{\mathrm{S}}^{\mathrm{N}}$

$$
M^{\mathrm{N}}=M_{0}^{\mathrm{N}}+M_{\mathrm{S}}^{\mathrm{N}}
$$

To calculate $M_{0}^{\mathrm{N}}$, we replace the distance $h_{\mathrm{S}}$ with the real lift $h$ of the two coils in (9).

\subsection{The Biot-Savart law}

In this section, we considered a multi turn coil, Fig. 4(a). The copper tracks of PCB coils were modelled as current filaments, in which each straight line segment is represented with a filament, positioned at the centre of the track, Fig. 4(b), [11]. The inner sides of coil $\mathrm{T}$ are $2 a_{1}$ and $2 b_{1}$.
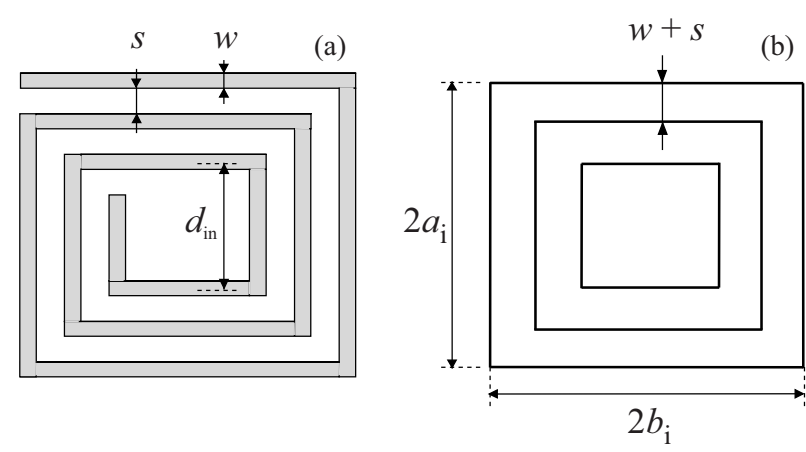

Fig. 4. (a) - spiral coil with three turns, (b) - filament single-turn coil

Figure 5 illustrates the one turn configuration of the coil. A differential magnetic induction, $\mathrm{d} \boldsymbol{B}_{\mathrm{d}_{x^{\prime}}}$ results from a differential current element, $I \mathrm{~d} x^{\prime}$, and exhibits the direction acquired through the cross product of $\mathbf{a}_{\mathrm{x}}$ and $\mathbf{a}_{\mathrm{R}}$.

The next relationship is given by the Biot-Savart law

$$
\mathrm{d} \boldsymbol{B}_{\mathrm{d} x^{\prime}}=\frac{\mu_{0} I}{4 \pi} \frac{\mathrm{d} x \mathbf{a}_{x} \times \mathbf{a}_{R}}{R_{\mathrm{d}}^{2}},
$$

where $\mu_{0}$ is the permeability of free space.

The distance $R_{\mathrm{d}}$ between the element $\mathrm{d} x^{\prime}$ of segment $\mathrm{CD}$ and the element of area $\mathrm{d} S_{j}$ of $R_{j}$ coil is

$$
R_{\mathrm{d}}=\sqrt{(b-y)^{2}+h_{\mathrm{S}}^{2}+\left(x^{\prime}-x\right)^{2}} .
$$

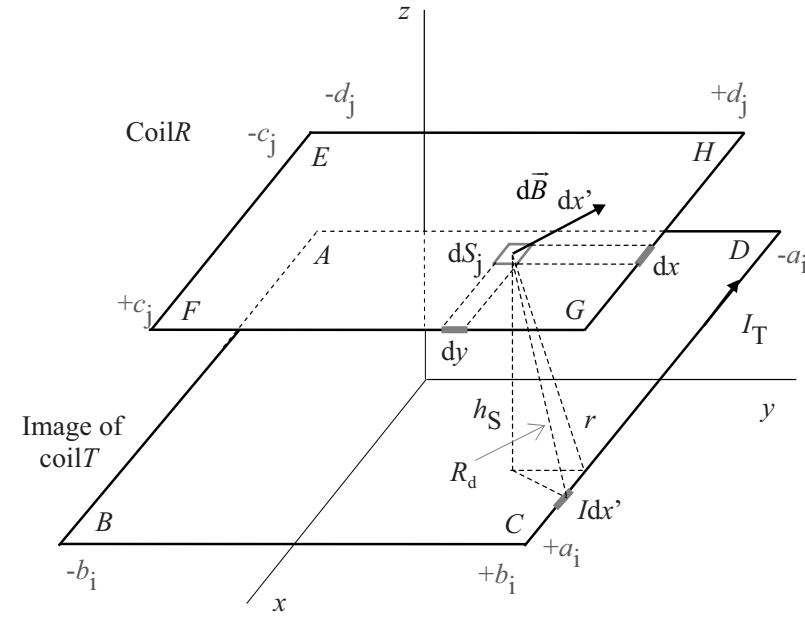

Fig. 5. Two coaxial single-turn filament rectangular coil with differential of induction vector $\boldsymbol{B}_{\mathrm{d} x^{\prime}}$ representation

The vector $\mathbf{a}_{\mathrm{R}}$ is calculated as follows

$$
\mathbf{a}_{\mathrm{R}}=\frac{1}{R_{\mathrm{d}}}\left[\left(x^{\prime}-x\right) \mathbf{a}_{x}+(b-y) \mathbf{a}_{y}+h_{\mathrm{S}} \mathbf{a}_{x}\right]
$$

and the cross product $\mathbf{a}_{\mathrm{x}}$ and $\mathbf{a}_{\mathrm{R}}$ is

$$
\mathbf{a}_{\mathrm{x}} \times \mathbf{a}_{\mathrm{R}}=\frac{1}{R_{\mathrm{d}}}\left[(b-y) \mathbf{a}_{x}+h_{\mathrm{S}} \mathbf{a}_{y}\right]=\sqrt{\frac{(b-y)^{2}+h_{\mathrm{S}}^{2}}{R_{\mathrm{d}}}} \mathbf{a}_{\emptyset}
$$

Thus, (11) becomes

$$
\mathrm{d} \boldsymbol{B}_{\mathrm{d} x^{\prime}}=\frac{\mu_{0} I}{4 \pi} \frac{\sqrt{(b-y)^{2}+h_{\mathrm{s}}^{2}}}{R_{\mathrm{d}}^{3}} \mathrm{~d} x^{\prime} .
$$

All the elements constituting the complete current filament in segment $\mathrm{CD}$ contribute to $B_{\mathrm{CD}}$ and must be included. Summation leads to the integral form of the Biot-Savart law

$$
\left[B_{i}\right]_{C D}=\frac{\mu_{0} I}{4 \pi} \int_{-a_{i}}^{+a_{i}} \frac{\sqrt{\left(b_{i}-y\right)^{2}+h_{\mathrm{S}}^{2}}}{\left.\left[b_{i}-y\right)^{2}+h_{\mathrm{S}}^{2}+\left(x^{\prime}-x\right)^{2}\right]^{3 / 2}} \mathrm{~d} x^{\prime} .
$$

This type of integral can be expressed as follow

$$
\begin{aligned}
{\left[B_{i}\right]_{C D} } & =\frac{\mu_{0} I}{4 \pi} \frac{1}{\sqrt{\left(b_{i}-y\right)^{2}+h_{\mathrm{S}}^{2}}} \\
& \times\left[\int_{-a_{i}}^{+a_{i}} \frac{\left[\sqrt{\left(b_{i}-y\right)^{2}+h_{\mathrm{S}}^{2}+\left(x^{\prime}-x\right)^{2}}\right]^{2}}{\left[\left(b_{i}-y\right)^{2}+h_{\mathrm{S}}^{2}+\left(x^{\prime}-x\right)^{2}\right]^{3 / 2}} \mathrm{~d} x^{\prime}\right. \\
& \left.-\int_{-a_{i}}^{+a_{i}} \frac{\left(x^{\prime}-x\right)^{2}}{\left[\left(b_{i}-y\right)^{2}+h_{\mathrm{S}}^{2}+\left(x^{\prime}-x\right)^{2}\right]^{3 / 2}} \mathrm{~d} x^{\prime}\right]
\end{aligned}
$$


to obtain

$$
\begin{aligned}
{\left[B_{i}\right]_{C D} } & =\frac{\mu_{0} I}{4 \pi} \frac{1}{\sqrt{\left(b_{i}-y\right)^{2}+h_{\mathrm{S}}^{2}}} \\
& \times\left[\int_{-a_{i}}^{+a_{i}} \frac{\sqrt{\left(b_{i}-y\right)^{2}+h_{\mathrm{S}}^{2}+\left(x^{\prime}-x\right)^{2}}}{\left[\sqrt{\left(b_{i}-y\right)^{2}+h_{\mathrm{S}}^{2}+\left(x^{\prime}-x\right)^{2}}\right]^{2}} \mathrm{~d} x^{\prime}\right. \\
& \left.-\int_{-a_{i}}^{+a_{i}} \frac{\left(x^{\prime}-x\right) \frac{2\left(x^{\prime}-x\right)}{2 \sqrt{\left(b_{i}-y\right)^{2}+h_{\mathrm{S}}^{2}+\left(x^{\prime}-x\right)^{2}}}}{\left[\sqrt{\left(b_{i}-y\right)^{2}+h_{\mathrm{S}}^{2}+\left(x^{\prime}-x\right)^{2}}\right]^{2}} \mathrm{~d} x^{\prime}\right] .
\end{aligned}
$$

Using the definition of the fraction derivative

$$
\begin{aligned}
{\left[B_{i}\right]_{C D} } & =\frac{\mu_{0} I}{4 \pi} \frac{1}{\sqrt{\left(b_{i}-y\right)^{2}+h_{\mathrm{S}}^{2}}} \\
& \times \int_{-a_{i}}^{+a_{i}}\left[\frac{\left(x^{\prime}-x\right)}{\sqrt{\left(b_{i}-y\right)^{2}+h_{\mathrm{S}}^{2}+\left(x^{\prime}-x\right)^{2}}}\right]^{\prime} \mathrm{d} x^{\prime} .
\end{aligned}
$$

Hence, we obtain

$$
\begin{aligned}
{\left[B_{i}\right]_{C D} } & =\frac{\mu_{0} I}{4 \pi} \frac{1}{\sqrt{\left(b_{i}-y\right)^{2}+h_{\mathrm{S}}^{2}}} \\
& \times\left[\frac{\left(a_{i}+x\right)}{\sqrt{\left(b_{i}-y\right)^{2}+h_{\mathrm{S}}^{2}+\left(a_{i}+x\right)^{2}}}\right. \\
& \left.+\frac{\left(a_{i}-x\right)}{\sqrt{\left(b_{i}-y\right)^{2}+h_{\mathrm{S}}^{2}+\left(a_{i}-x\right)^{2}}}\right] .
\end{aligned}
$$

The $z$-axis component of $B_{\mathrm{CD}}$ is $B_{\mathrm{CD} . \mathrm{z}}$. The two cases in our induction calculation are distinguished:

- $b_{i} \geq d_{j}$.

The width $b_{i}$ of coil $\mathrm{T}$ is superior or equal to the width $d_{j}$ of coil R; in this case, all the $z$-axis components of the vectors of induction $B$ are positive in all areas $S_{j}$, Fig. 6.

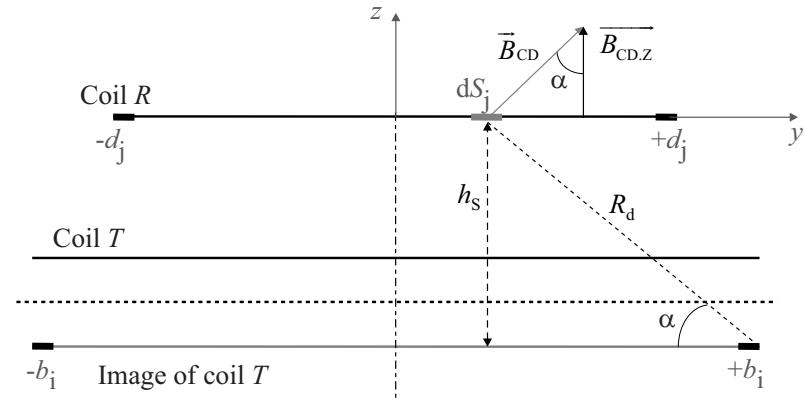

Fig. 6. Induction vector when $b_{i} \geq d_{j}$

Since $\cos \alpha=\frac{b_{i}-y}{\sqrt{\left(b_{i}-y\right)^{2}+h_{\mathrm{S}}^{2}}}$, we have

$$
\left[\Phi_{i j}\right]_{C D}=\frac{\mu_{0} I}{4 \pi} \int_{-d_{j}}^{+d_{j}} \int_{-c_{j}}^{+c_{j}}\left[B_{i j}\right]_{C D} \cos \alpha \mathrm{d} x \mathrm{~d} y .
$$

- $b_{i}<d_{j}$

The width $b_{i}$ of coil $\mathrm{T}$ is inferior to the width $d_{j}$ of coil R; in this case, the components of the vectors of induction $B$ are positive $\left(B_{\mathrm{CD} . Z .1}\right)$, when $y$ varies from $d_{j}$ to $+b_{i}$; the calculation of $\Phi_{\mathrm{CD}}$ remains the same as shown in the first case, and when $y$ varies from $+b_{i}$ to $+d_{j}$, other $z$-axis components become negative $\left(B_{\mathrm{CD} . Z .2}\right)$, Fig. 7.

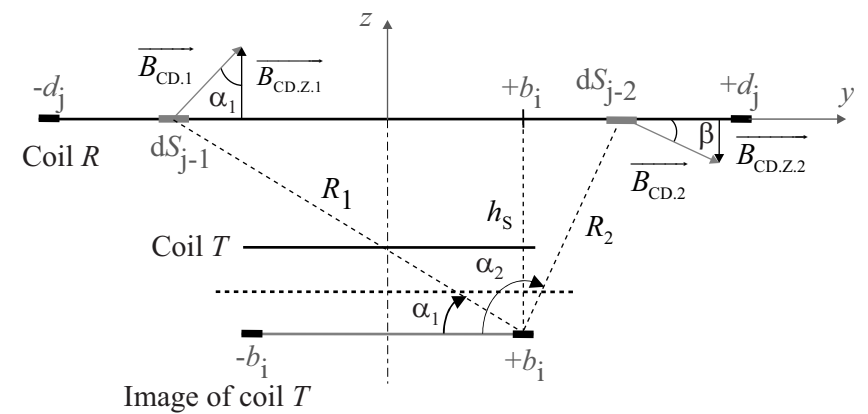

Fig. 7. Induction vector when $b_{i}<d_{j}$

$B_{\mathrm{CD} . \mathrm{Z} .1}=B_{\mathrm{CD} .1} \cos \alpha_{1}$, and $B_{\mathrm{CD} . \mathrm{Z} .2}=B_{\mathrm{CD} .2} \sin \beta$.

The calculus of flux $\Phi_{\mathrm{CD}}$ is equal to the difference between the positive and negative parts.

$$
\begin{aligned}
\Phi_{\mathrm{CD}}=\frac{\mu_{0} I}{4 \pi} \int_{-d_{j}}^{+b_{i}} \int_{-c_{j}}^{+c_{j}}\left[B_{i j}\right]_{C D .1} \cos \alpha_{1} \mathrm{~d} x \mathrm{~d} y \\
-\frac{\mu_{0} I}{4 \pi} \int_{-b_{i}}^{+d_{j}} \int_{-c_{j}}^{+c_{j}}\left[B_{i j}\right]_{C D .2} \sin \beta \mathrm{d} x \mathrm{~d} y .
\end{aligned}
$$

Since $\sin \beta=-\cos \alpha_{2}=\frac{y-b_{i}}{\sqrt{\left(b_{i}-y\right)^{2}+h_{\mathrm{S}}^{2}}}$ we have

$$
\begin{aligned}
{\left[\Phi_{i j}\right]_{\mathrm{CD}} } & =\frac{\mu_{0} I}{4 \pi} \int_{-d_{j}}^{+b_{i}} \int_{-c_{j}}^{+c_{j}}\left[B_{i j}\right]_{\mathrm{CD} .1} \cos \alpha_{1} \mathrm{~d} x \mathrm{~d} y \\
& +\frac{\mu_{0} I}{4 \pi} \int_{-b_{i}}^{+d_{j}} \int_{-c_{j}}^{+c_{j}}\left[B_{i j}\right]_{\mathrm{CD} .2} \cos \alpha_{2} \mathrm{~d} x
\end{aligned}
$$

and

$$
\oint B_{\mathrm{CD}} \mathrm{d} S=\oint B_{\mathrm{CD} .1} \mathrm{~d} S_{1}+\oint B_{\mathrm{CD} .2} \mathrm{~d} S_{2} .
$$

Hence,

$$
\left[\Phi_{i j}\right]_{\mathrm{CD}}=\frac{\mu_{0} I}{4 \pi} \int_{-d_{j}}^{+d_{j}} \int_{-c_{j}}^{+c_{j}}\left[B_{i j}\right]_{\mathrm{CD}} \cos \alpha \mathrm{d} x \mathrm{~d} y .
$$

Finally, the same integral is obtained for the two cases, and the same solution must be determined. The next step is to calculate the following integral

$$
A_{i j}=\frac{\mu_{0} I}{4 \pi} \int_{-c_{j}}^{+c_{j}}\left[B_{i j}\right]_{\mathrm{CD}} \cos \alpha \mathrm{d} x .
$$


Then

$$
\begin{aligned}
A_{i j} & =\frac{\mu_{0} I}{4 \pi} \frac{b_{i}-y}{\left(b_{i}-y\right)^{2}+h_{\mathrm{S}}^{2}} \\
& \times \int_{-c_{j}}^{+c_{j}}\left[\frac{a_{i}+x}{\sqrt{\left(b_{i}-y\right)^{2}+h_{\mathrm{S}}^{2}+\left(a_{i}+x\right)^{2}}}\right. \\
& \left.+\int_{-c_{j}}^{+c_{j}} \frac{a_{i}-x}{\sqrt{\left(b_{i}-y\right)^{2}+h_{\mathrm{S}}^{2}+\left(a_{i}-x\right)^{2}}}\right] \mathrm{d} x .
\end{aligned}
$$

Thus, according to the integration we have

$$
\begin{aligned}
A_{i j} & =\frac{\mu_{0} I}{4 \pi} \frac{b_{i}-y}{\left(b_{i}-y\right)^{2}+h_{\mathrm{S}}^{2}} \\
& \times\left(\left[\sqrt{\left(b_{i}-y\right)^{2}+h_{\mathrm{S}}^{2}+\left(a_{i}+x\right)^{2}}\right]_{-c_{j}}^{+c_{j}}\right. \\
& \left.-\left[\sqrt{\left(b_{i}-y\right)^{2}+h_{\mathrm{S}}^{2}+\left(a_{i}-x\right)^{2}}\right]_{-c_{j}}^{+c_{j}}\right)
\end{aligned}
$$

implying

$$
\begin{aligned}
A_{i j} & =\frac{\mu_{0} I}{2 \pi} \frac{b_{i}-y}{\left(b_{i}-y\right)^{2}+h_{\mathrm{S}}^{2}} \\
& \times\left[\sqrt{\left(b_{i}-y\right)^{2}+h_{\mathrm{S}}^{2}+\left(a_{i}+c_{j}\right)^{2}}\right. \\
& \left.-\sqrt{\left(b_{i}-y\right)^{2}+h_{\mathrm{S}}^{2}+\left(a_{i}-c_{j}\right)^{2}}\right] .
\end{aligned}
$$

Finally, the calculus of magnetic flux $\Phi_{\mathrm{CD}}$ is given using the integral

$$
\begin{aligned}
& {\left[\Phi_{i j}\right]_{\mathrm{CD}}=\int_{-d_{j}}^{+d_{j}} A_{i j} \mathrm{~d} y=} \\
& \frac{\mu_{0} I}{2 \pi} \int_{-d_{j}}^{+d_{j}} \frac{b_{i}-y}{\left(b_{i}-y\right)^{2}+h_{\mathrm{S}}^{2}}\left[\sqrt{\left(b_{i}-y\right)^{2}+h_{\mathrm{S}}^{2}+\left(a_{i}+c_{j}\right)^{2}}-\right. \\
& \left.\sqrt{\left(b_{i}-y\right)^{2}+h_{\mathrm{S}}^{2}+\left(a_{i}-c_{j}\right)^{2}}\right] \mathrm{d} y . \quad
\end{aligned}
$$

By substituting $b_{i}-y=y^{\prime}$, we have for $y=-d_{j} \Rightarrow y^{\prime}=$ $b_{i}+d_{j}$ and for $y=+d_{j} \Rightarrow y^{\prime}=b_{i}-d_{j}$, then we obtain

$$
\begin{array}{r}
{\left[\Phi_{i j}\right]_{\mathrm{CD}}=\frac{\mu_{0} I}{2 \pi} \int_{b_{i}-d_{j}}^{b_{i}+d_{j}} \frac{y^{\prime}}{y^{\prime 2}+h_{\mathrm{S}}^{2}}\left(\sqrt{y^{\prime 2}+h_{\mathrm{S}}^{2}+\left(a_{i}+c_{j}\right)^{2}}-\right.} \\
\left.\sqrt{y^{\prime} 2+h_{\mathrm{S}}^{2}+\left(a_{i}-c_{j}\right)^{2}}\right)
\end{array}
$$

The solution of this type of integral, $K$, which is used in subsequent calculus, is as follows

$$
K=\int \frac{p}{p^{2}+z^{2}} \sqrt{p^{2}+y^{2}+m^{2}} \mathrm{~d} p .
$$

By substituting $q$ with $p$, we obtain $q^{2}=p^{2}+z^{2}+m^{2} \Longrightarrow q=\sqrt{p^{2}+z^{2}+m^{2}}$, $q \mathrm{~d} q=p \mathrm{~d} p \Longrightarrow \mathrm{d} p=\frac{q}{p} \mathrm{~d} q$, $p^{2}+z^{2}=q^{2}-m^{2} \Longrightarrow p=\sqrt{q^{2}-z^{2}-m^{2}}$.

Then

$$
\begin{gathered}
K=\int \frac{\sqrt{q^{2}-z^{2}-m^{2}}}{q^{2}-m^{2}} q \frac{q}{\sqrt{q^{2}-z^{2}-m^{2}}} \mathrm{~d} q \\
=\int \frac{q^{2}}{q^{2}-m^{2}} \mathrm{~d} q=\int \mathrm{d} q+m^{2} \int \frac{\mathrm{d} q}{q^{2}-m^{2}} .
\end{gathered}
$$

Thus, we have

$$
K=\int \mathrm{d} q+\int \frac{\mathrm{d} q}{\left(\frac{q}{m}\right)^{2}-1} .
$$

By this transformation, we obtain

$$
k=\frac{q}{m} \Longrightarrow \mathrm{d} k=\frac{1}{m} \mathrm{~d} q \Longrightarrow \mathrm{d} q=m \mathrm{~d} k .
$$

Hence,

$$
\begin{aligned}
K=q+\int \frac{m \mathrm{~d} k}{k^{2}-1}=q-m \int & \frac{\mathrm{d} k}{1-k^{2}} \\
& =q-m \tanh ^{-1} k+C^{s t} .
\end{aligned}
$$

Finally, this integral is

$$
\begin{aligned}
K & =\sqrt{p^{2}+z^{2}+m^{2}}-m \tanh ^{-1} \frac{\sqrt{p^{2}+z^{2}+m^{2}}}{m} \\
& =\sqrt{p^{2}+z^{2}+m^{2}}-m \tanh ^{-1} \frac{m}{\sqrt{p^{2}+z^{2}+m^{2}}}
\end{aligned}
$$

From (21) and (22), $\Phi_{\mathrm{CD}}$ becomes

$$
\begin{gathered}
{\left[\Phi_{i j}\right]_{\mathrm{CD}}=\frac{\mu_{0} I}{2 \pi} \times\left(\left[\sqrt{y^{\prime 2}+h_{\mathrm{S}}^{2}+\left(a_{i}+c_{j}\right)^{2}}\right]_{b_{i}-d_{j}}^{b_{i}+d_{j}}\right.} \\
-\left[\left(a_{i}+c_{j}\right) \tanh ^{-1} \frac{a_{i}+c_{j}}{\sqrt{y^{\prime 2}+h_{\mathrm{S}}^{2}+\left(a_{i}+c_{j}\right)^{2}}}\right]_{b_{i}-d_{j}}^{b_{i}+d_{j}} \\
-\left[\sqrt{y^{\prime 2}+h_{\mathrm{S}}^{2}+\left(a_{i}+c_{j}\right)^{2}}\right]_{b_{i}-d_{j}}^{b_{i}+d_{j}} \\
\left.+\left[\left(a_{i}+c_{j}\right) \tanh ^{-1} \frac{a_{i}+c_{j}}{\sqrt{y^{\prime 2}+h_{\mathrm{S}}^{2}+\left(a_{i}+c_{j}\right)^{2}}}\right]_{b_{i}-d_{j}}^{b_{i}+d_{j}}\right)
\end{gathered}
$$

Thus, we obtain

$$
\begin{aligned}
{\left[\Phi_{i j}\right]_{\mathrm{CD}} } & =\frac{\mu_{0} I}{2 \pi}\left[\sqrt{\left(b_{i}+d_{j}\right)^{2}+h_{\mathrm{S}}^{2}+\left(a_{i}+c_{j}\right)^{2}}\right. \\
& -\sqrt{\left(b_{i}-d_{j}\right)^{2}+h_{\mathrm{S}}^{2}+\left(a_{i}+c_{j}\right)^{2}} \\
& -\left(a_{i}+c_{j}\right) \tanh ^{-1} \frac{a_{i}+c_{j}}{\sqrt{\left(b_{i}+d_{j}\right)^{2}+h_{\mathrm{S}}^{2}+\left(a_{i}+c_{j}\right)^{2}}} \\
& +\left(a_{i}+c_{j}\right) \tanh ^{-1} \frac{a_{i}+c_{j}}{\sqrt{\left(b_{i}-d_{j}\right)^{2}+h_{\mathrm{S}}^{2}+\left(a_{i}+c_{j}\right)^{2}}} \\
& -\sqrt{\left(b_{i}+d_{j}\right)^{2}+h_{\mathrm{S}}^{2}+\left(a_{i}-c_{j}\right)^{2}}
\end{aligned}
$$




$$
\begin{gathered}
+\sqrt{\left(b_{i}-d_{j}\right)^{2}+h_{\mathrm{S}}^{2}+\left(a_{i}-c_{j}\right)^{2}} \\
+\left(a_{i}-c_{j}\right) \tanh ^{-1} \frac{a_{i}-c_{j}}{\sqrt{\left(b_{i}+d_{j}\right)^{2}+h_{\mathrm{S}}^{2}+\left(a_{i}-c_{j}\right)^{2}}} \\
-\left(a_{i}-c_{j}\right) \tanh ^{-1} \frac{a_{i}-c_{j}}{\sqrt{\left(b_{i}-d_{j}\right)^{2}+h_{\mathrm{S}}^{2}+\left(a_{i}-c_{j}\right)^{2}}},
\end{gathered}
$$

where $\Phi_{\mathrm{CD}}$ is the effective magnetic flux produced by segment $\mathrm{CD}$. The rectangle length is the same for segment $\mathrm{AB}$ as that for segment $\mathrm{CD}$. For the rectangle width ( $\mathrm{BC}$ and $\mathrm{AD}$ ), we can use (24) to calculate the magnetic flux.

The relation between the magnetic flux and the mutual inductance is

$$
\Phi=M I
$$

If the current value of $I$ in the coil is equal to the unit, all segment parts are equal to the corresponding magnetic flux calculated

$$
\begin{gathered}
{\left[\Phi_{i j}\right]_{\mathrm{CD}}=\left[\Phi_{i j}\right]_{\mathrm{AB}}=M_{i j}^{\mathrm{l}} I} \\
{\left[\Phi_{i j}\right]_{\mathrm{AD}}=\left[\Phi_{i j}\right]_{\mathrm{BC}}=M_{i j}^{\mathrm{w}} I .}
\end{gathered}
$$

Hence, the global mutual inductance calculated using the Biot-Savart method is

$$
M_{\mathrm{S}}^{\mathrm{BS}}=2 \sum_{i=1}^{N_{T}} \sum_{j=1}^{N_{R}}\left[M_{i j}^{1}+M_{i j}^{\mathrm{w}}\right],
$$

where, $M_{i j}^{1}$ is the mutual inductance between length segments $\mathrm{CD}, \mathrm{AB}$ and $\mathrm{GH}, \mathrm{EF}$, and. $M_{i j}^{\mathrm{w}}$ is the mutual inductance between width segments $\mathrm{AD}, \mathrm{BC}$ and FG, EH.

Finally the global mutual inductance $M^{\mathrm{BS}}$, with BiotSavart low, is equal to the sum of the mutual inductance without magnetic layer $M_{0}^{\mathrm{BS}}$, and the mutual inductance of the image of T-coil with R-coil $M_{\mathrm{S}}^{\mathrm{BS}}$

$$
M^{\mathrm{BS}}=M_{0}^{\mathrm{BS}}+M_{\mathrm{S}}^{\mathrm{BS}}
$$

To calculate of $M_{0}^{\mathrm{N}}$, the distance $h_{\mathrm{S}}$ is replaced with the real lift $h$ between two coils in (26).

\section{Measurement and analytical calculation}

The mutual inductance was calculated by implementing a code representation of Neumann and Biot-Savart methods as an algorithm in a computer program. The analytical solution for calculating the mutual inductance between two multi-turn spiral inductors is acquired from (10) and (27). With the analytical solution, the defined integrals can be calculated with a low computational effort to evaluate two coaxial-positioned filamentary structures.

To measure the mutual inductance of two similar PCB coils, we used a low-frequency LEADER-745 LCR-meter.
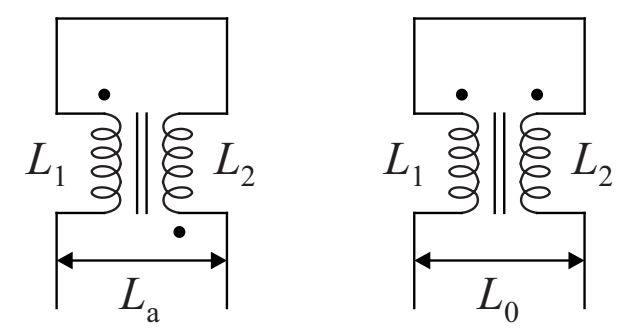

Fig. 8. Mutual inductance measurement using series aiding and series opposing techniques

The mutual inductance value was directly obtained from the inductance measurement by connecting the two coils (Fig. 8). When test current $I$ flows through primary winding, the secondary voltage is $V=j \omega M I$. Therefore, the mutual inductance can be calculated from the ratio of the secondary voltage $V$ to primary current $I$. However, the applicable frequency range of both the measurement techniques is limited by the type and parameter values of the coils measured. In these methods, stray capacitance effects, including the distributed capacitance of windings, inter-winding capacitance, and test lead capacitance, are considered sufficiently small [12].

Different sets of inductors were fabricated using a commercial one-layer PCB with FR-4 as the substrate material. We acquired three pairs of coils with 5,10 , and 15 turns. The effects of the number of turns, with and without magnetic layer is described in the subsequent subsections.

\subsection{Mutual inductance without magnetic layer}

In this case, we set all the geometrical parameters values, such as spacing $\left(s_{\mathrm{T}}=s_{\mathrm{R}}=0.5 \mathrm{~mm}\right)$, track width $\left(w_{\mathrm{T}}=w_{\mathrm{R}}=0.6 \mathrm{~mm}\right)$, and length and width of $\mathrm{T}$ and $\mathrm{R}$ coils, calculated the mutual inductance, and determined the function of the number of turns. The dimension of the internal side of square coils $\mathrm{T}$ and $\mathrm{R}$ was $14 \mathrm{~mm}$, and the external side dimensions of square coils were $22.8,33.8$, and $44.8 \mathrm{~mm}$ for 5, 10, and 15turns, respectively.

The calculation and measurement results are presented in Tab. 1 and Fig. 9. Discrepancy is denoted Discr., Measurement: Meas., Neumann method: N, Biot-Savart method: BS.

With an increase in the number of turns, the values of mutual inductance calculated using both the methods increase. Initially, these values are different. The mutual inductance value calculated with the Biot-Savart method is higher than to that calculated with the Neumann method, especially between 0 and $6 \mathrm{~mm}$; however, when the lift, $h$, increases, the mutual inductance acquired using the two methods converges. Thus, the results acquired from the Biot-Savart method are closer to the experimental measurements than those obtained from the Neumann method. 

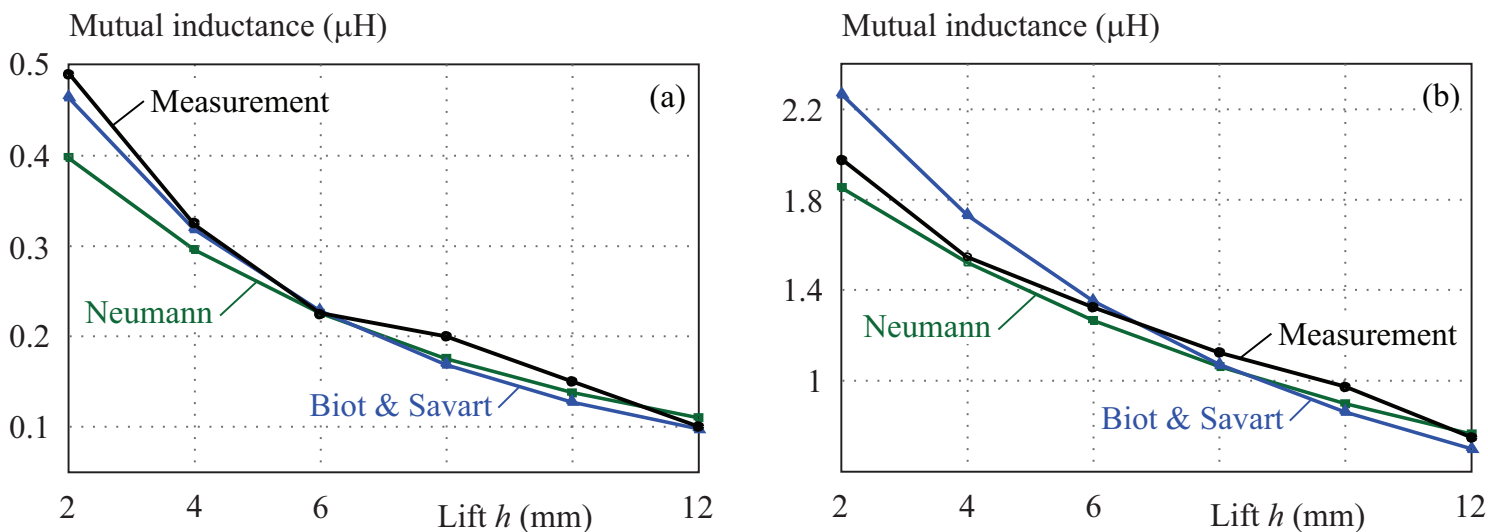

Mutual inductance $(\mu \mathrm{H})$

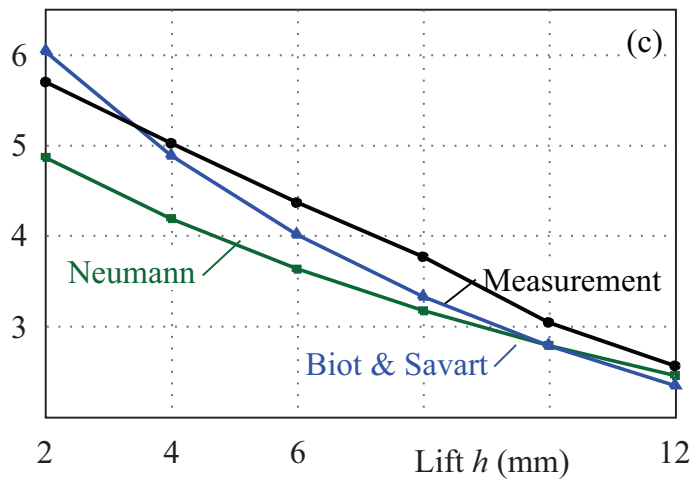

Fig. 9. Mutual inductance, function number of turns without magnetic Layer: (a) - 5, (b) - 10, and (c) - 15 turn coils
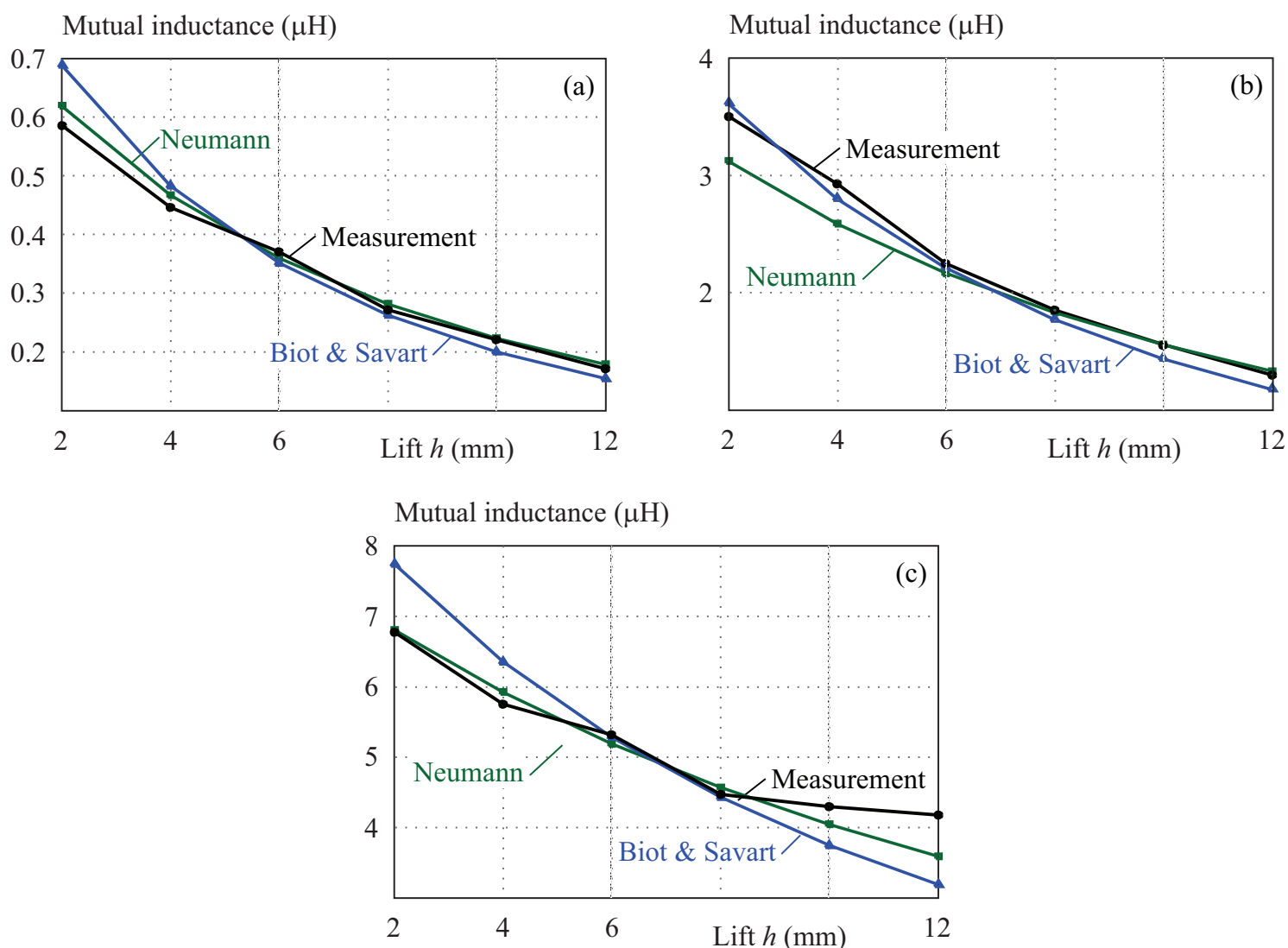

Fig. 10. Mutual inductance, function number of turns with magnetic Layer: (a) - 5, (b) - 10, and (c) - 15 turn coils 
Table 1. Mutual inductance measurement and analytical calculation without magnetic layer, for different lifts $(h)$

\begin{tabular}{|c|c|c|c|c|c|}
\hline $\begin{array}{l}h \\
(\mathrm{~mm})\end{array}$ & $\begin{array}{c}M_{\text {Meas }} \\
(\mu \mathrm{H})\end{array}$ & $\begin{array}{c}M_{N} \\
(\mu \mathrm{H})\end{array}$ & $\begin{array}{c}\text { Discr. (\%) } \\
\text { Meas-N }\end{array}$ & $\begin{array}{l}M_{\mathrm{BS}} \\
(\mu \mathrm{H})\end{array}$ & $\begin{array}{c}\text { Discr. (\%) } \\
\text { Meas-BS }\end{array}$ \\
\hline \multicolumn{6}{|c|}{5 turns } \\
\hline 2 & 0.490 & 0.397 & 18.9 & 0.464 & 5.3 \\
\hline 4 & 0.325 & 0.295 & 9.2 & 0.318 & 9.2 \\
\hline 6 & 0.225 & 0.226 & 0.4 & 0.228 & 1.3 \\
\hline 8 & 0.200 & 0.175 & 12.5 & 0.168 & 16.0 \\
\hline 10 & 0.150 & 0.138 & 8.0 & 0.127 & 15.3 \\
\hline 12 & 0.100 & 0.110 & 10.0 & 0.097 & 3.0 \\
\hline \multicolumn{6}{|c|}{10 turns } \\
\hline 2 & 1.975 & 1.853 & 6.2 & 2.266 & 14.7 \\
\hline 4 & 1.550 & 1.522 & 1.8 & 1.731 & 11.6 \\
\hline 6 & 1.325 & 1.266 & 4.5 & 1.352 & 2.0 \\
\hline 8 & 1.125 & 1.063 & 5.5 & 1.073 & 4.6 \\
\hline 10 & 0.975 & 0.899 & 7.8 & 0.862 & 11.5 \\
\hline 12 & 0.750 & 0.765 & 2.0 & 0.700 & 6.6 \\
\hline \multicolumn{6}{|c|}{15 turns } \\
\hline 2 & 5.700 & 4.869 & 14.5 & 6.040 & 5.9 \\
\hline 4 & 5.025 & 4.194 & 16.5 & 4.892 & 2.6 \\
\hline 6 & 4.375 & 3.642 & 16.7 & 4.019 & 8.1 \\
\hline 8 & 3.775 & 3.183 & 15.6 & 3.336 & 11.6 \\
\hline 10 & 3.050 & 2.796 & 8.3 & 2.793 & 8.4 \\
\hline 12 & 2.575 & 2.466 & 4.2 & 2.356 & 8.5 \\
\hline
\end{tabular}

\subsection{Mutual inductance with magnetic layer}

We set all the parameters with the same coils $\left(N_{\mathrm{T}}=\right.$ $N_{\mathrm{R}}=5,10,15$ turns), ie, same spacing values, track width and dimensions of internal and external side. A ferromagnetic layer is introduced under the coil $\mathrm{T}$, at a distance of $d_{0}=3 \mathrm{~mm}$

The calculation and measurement results are presented in Tab. 2 and Fig. 10.

In this configuration, the mutual inductance values calculated with the Biot-Savart method is higher than to that calculated with the Neumann method for reduced lift, then the values converge for lifts superior to $4 \mathrm{~mm}$. The measurements values are closer to values of Biot-Savart values for lifts inferior to $4 \mathrm{~mm}$, except for 15 turns. Then, The measurements values are closer to values of Neumann values for lifts superior to $4 \mathrm{~mm}$.

We also see, as expected, that the values of mutual inductance obtained by introducing a ferromagnetic layer, are much larger than the values of the configuration without magnetic layer.

\section{Conclusions}

In this work, two magnetostatic analytical models were presented and compared for calculating the mutual induc-
Table 2. Mutual inductance measurement and analytical calculation with magnetic layer, for different lifts $(h)$

\begin{tabular}{|c|c|c|c|c|c|}
\hline $\begin{array}{l}h \\
(\mathrm{~mm})\end{array}$ & $\begin{array}{c}M_{\text {Meas }} \\
(\mu \mathrm{H})\end{array}$ & $\begin{array}{c}M_{N} \\
(\mu \mathrm{H})\end{array}$ & $\begin{array}{c}\text { Discr. (\%) } \\
\text { Meas-N }\end{array}$ & $\begin{array}{l}M_{\mathrm{BS}} \\
(\mu \mathrm{H})\end{array}$ & $\begin{array}{c}\text { Discr. (\%) } \\
\text { Meas-BS }\end{array}$ \\
\hline \multicolumn{6}{|c|}{5 turns } \\
\hline 2 & 0.590 & 0.623 & 5.6 & 0.692 & 17.2 \\
\hline 4 & 0.450 & 0.471 & 4.6 & 0.486 & 8.0 \\
\hline 6 & 0.375 & 0.363 & 3.2 & 0.355 & 5.3 \\
\hline 8 & 0.275 & 0.285 & 3.6 & 0.266 & 3.2 \\
\hline 10 & 0.225 & 0.229 & 1.7 & 0.203 & 9.8 \\
\hline 12 & 0.175 & 0.182 & 4.0 & 0.158 & 9.7 \\
\hline \multicolumn{6}{|c|}{10 turns } \\
\hline 4 & 2.925 & 2.586 & 11.5 & 2.804 & 4.1 \\
\hline 6 & 2.250 & 2.166 & 3.7 & 2.214 & 1.6 \\
\hline 8 & 1.850 & 1.829 & 1.1 & 1.773 & 4.2 \\
\hline 10 & 1.550 & 1.555 & 0.3 & 1.437 & 7.3 \\
\hline 12 & 1.300 & 1.330 & 2.3 & 1.176 & 9.5 \\
\hline \multicolumn{6}{|c|}{15 turns } \\
\hline 2 & 6.775 & 6.811 & 0.5 & 7.748 & 14.3 \\
\hline 4 & 5.750 & 5.925 & 3.0 & 6.358 & 10.5 \\
\hline 6 & 5.325 & 5.191 & 2.5 & 5.284 & 0.7 \\
\hline 8 & 4.475 & 4.572 & 2.1 & 4.433 & 0.9 \\
\hline 10 & 4.300 & 4.044 & 5.9 & 3.749 & 12.8 \\
\hline 12 & 4.175 & 3.592 & 13.9 & 3.191 & 23.5 \\
\hline
\end{tabular}

tance between two coaxial PCB inductors incorporating ferromagnetic layer. The mutual inductance was calculated by implementing the analytical representation of the Neumann formula and Biot-Savart law as an algorithm in the computer program. The results of the two methods were compared with the experimental results, and a strong agreement was observed for several parameters; lift and number of turns, of planar PCB coils. This study provided two generalised analytical formulas, with complete demonstration. The mutual inductance can be calculated analytically with a low computational effort, within fractions of a second. These models can be used for fast estimation of mutual inductances of rectangular spiral coils embedded with magnetic layer.

\section{Acknowledgements}

This study was supported by the General Directorate of Scientific Research and Technological Development (DGRSDT) of Algeria. We would also like to thank the anonymous reviewer for his valuable advice.

\section{REFERENCES}

[1] A. B. Islam, S. K. Islam, and F. S. Tulip, "Design and Optimization of Printed Circuit Board Inductors for Wireless Power 
Transfer System", Journal of Scientific Research Publishing, 2013, https://doi.org/10.4236/cs.2013.42032.

[2] G. Vigneau, M. Cheikh, R. Benbouhout, and A. Takacs, "Design and modeling of PCB coils for inductive power charging", Wireless Power Transfer, Cambridge University Press, vol. 2, no 2, pp. 143-152, 2005, https://doi.org/10.1017/wpt.2015.17.

[3] F. Durmus and S. Karagol, "Mutual Inductance Calculation Formula for Planar Square Coils", IEEE 2nd International Symposium on Multidisciplinary Studies and Innovative Technologies, (ISMSIT), 2018 https://doi.org/10.1109/ISMSIT.2018.8567040.

[4] M. Zhang, M. R. Haider, M. A. Huque, M. A. Adeeb, S. R. Rahman, and S. K. Islam, "A Low Power Sensor Signal Processing Circuit for Implantable Biosensor Applications", Smart Materials \& Structures, vol. 16, no. 2, pp. 525-530, 2007, https://dx.doi.org/10.10 88/0964-1726/16/2/034.

[5] S. Pinhas, S. W. Hwang, and J. S. Rieh, "A Magnetostatic Model for Square Spiral Inductors Incorporating a Magnetic Layer", IEEE Transaction Magnetics, vol. 44, no. 8, pp. 2085-2087, August 2008, https://doi.org/10.1109/TMAG.2008.923482.

[6] C. Akyel, S. Babic, and E. Skaljo, "A New Analytical Model for Square Spiral Inductors Incorporating a Magnetic Layer", Proceedings of the Asia-Pacific Microwave Conference, 2011.http://dx.doi.org/10.13140/2.1.2871.2962.

[7] H. A. Haus and J. R. Melcher, Electromagnetic Fields and Energy, Massachusetts Institute of Technology, pp. 310-322.

[8] F. W. Grover, Inductance Calculations, 1964, New York: Dover.

[9] C. L. W. Sonntag, E. A. Lomonova, and J. L. Duarte, "Implementation of the Neumann Formula for Calculating the Mutual Inductance between Planar PCB Inductors", IEEE, Proceedings of the 18th International Conference on Electrical Machines, 2008, https://doi.org/10.1109/ICELMACH.2008.4799978.

[10] E. B. Rosa, "The Self and Mutual Inductances of Linear Conductors", Bulletin of the Bureau of Standards, vol. , no. 2, pp. 301-344, 1908, https://dx.doi.org/10.6028/bulletin.088.

[11] Y. Cheng and Y. Shu, "A New Analytical Calculation of the Mutual Inductance of the Coaxial Spiral Rectangular Coils", IEEE, Transactions on Magnetics, vol. 50, no. 4, 2014, https://doi.org/10.1109 /TMAG.2013.2290972.

[12] Agilent Technologies, Impedance Measurement Handbook, A Guide to Measurement technology and techniques, 4th Edition, 2009.

Received 27 July 2021

Salah-Eddine Bendimerad was born in Sidi Bel-Abbes, Algeria, in 1973. He received the Engineer and the Magister
(Dr-Eng) degrees in electrical engineering from Djillali Liabes University of Sidi Bel-Abbes, Algeria, in 1995 and 2007 respectively. Since 2012 he is an Assistant Professor at Djillali Liabes University of Sidi Bel-Abbes, Algeria. He got his Ph.D. degree in high-voltage in 2013 and became in 2014 Lecturer at the same University. His research interests include electrostatic and electromagnetic applications.

Selma Baghli was born in Algiers, Algeria, in 1975. She received the Superior Diploma of Mathematics and the Magister degree on Mathematical Analysis at the Abou bekr Belkaid University of Tlemcen, Algeria, in 1995 and 1998 respectively, and in 2008 she got the Ph.D. degree of Mathematics on Differential equations at the Djillali Liabes University. Since 2016 she is a Full Professor of Mathematics at the same university. Her research interests are in ordinary and partial differential equations and numerical analysis.

Abdelghani Ayad was born in Algeria, in 1969. He received a PhD degree at the Djillali Liabes University of Sidi Bel-Abbes in 2009 in Electrical Engineering specializing in electromagnetic and NDT by eddy current. He is currently working at the Djillali Liabes University of Sidi Bel-Abbes, Department of Electrical Engineering as a Full Professor. His research interest is particularly about the characterization of materials by eddy current and electromagnetic applications.

Amar Tilmatine received the MS degree in electrical engineering and the Magister (Dr Eng) degree from the University of Science and Technology, Oran, Algeria, in 1988 and 1991, respectively. He obtained the Doctorate degree in 2004 from the Electrical Engineering Institute of Sidi-Bel-Abbes University, Algeria. In 2010, he was promoted to the rank of Professor. He is also a Senior IEEE member and past-chair of IEEE Algeria section. Since 1991, he has been teaching electric field theory, high-voltage Engineering and applied electrostatics at the Institute of Electrical Engineering, Djillali Liabes University, Sidi-Bel-Abbes, Algeria. He is currently the director of the APELEC laboratory and the manager of the technological platform Industrial technologies situated in the Faculty of Technology at Djillali Liabes university. He published over 120 articles in refereed journals and 10 patents, with an $\mathrm{h}$ index 22. His research topics are related to high voltage applications, the main domain being the applied electrostatics, in particular electrostatic separators and precipitators. 